\title{
Multiples Myelom \\ Patienten profitieren von frühzeitiger und anhaltender Therapie mit Zoledronat
}

\begin{abstract}
Bei den meisten Patienten mit multiplem Myelom liegen schon bei der Diagnose Knochenschäden vor. Durch Bisphosphonate lässt sich die myelombedingte Zerstörung des Knochens vermindern. Dabei hat sich insbesondere Zoledronat als wirksam erwiesen.
\end{abstract}

\begin{abstract}
Die im Knochenmark angesiedelten Myelomzellen stehen über Zytokine in Wechselwirkung mit den übrigen dort befindlichen Zellen und bewirken eine Aktivierung der Osteoklasten und eine Hemmung der Osteoblasten. Dadurch entstehen die typischen Knochenläsionen. Durch Bisphosphonate, die sich in die mineralisierte Knochenmatrix einlagern, wird die osteoklastäre Knochenresorption vermindert. Beim multiplen Myelom sind Clodronat, Pamidronat und Zoledronat für eine knochenschützende Behandlung zugelassen, so Prof. Dr. Hartmut Goldschmidt, Heidelberg. In mehreren placebokontrollierten Studien zeigten sie eine schmerzreduzierende Wirkung und verringerten das weitere Auftreten von Skelettkomplikationen (SRE).
\end{abstract}

\section{Vorteile durch Zoledronat}

In der MRC-Myeloma-IX-Studie wurde Zoledronat (Zometa ${ }^{\circledast}$ ) mit Clodronat verglichen: Patienten mit neu diagnostiziertem multiplem Myelom erhielten zusätzlich zu intensiver oder nicht-intensiver Chemotherapie Zoledronat (4mg i.v. alle 3-4 Wochen; $\mathrm{n}=981$ ) oder Clodronat $(1.600 \mathrm{mg} / \mathrm{Tag}$ oral; $\mathrm{n}=979$ ) bis zur Progression (Morgan $\mathrm{G}$ et al., 2010, Lancet 376: 1989-1999). Nach einem medianen Follow-up von 3,7 Jahren betrug das mediane Gesamtüberleben unter Zoledronat 50 Monate versus 44,5 Monate unter Clodronat $(p=0,04)$. Der Überlebensvorteil durch Zoledronat war bereits nach vier Monaten signifikant $(p=0,004)$, was für einen frühzeitigen Einsatz des Bisphosphonats spricht. Über den gesamten Therapiezeitraum traten SRE im Zoledronat-Arm in geringerem Maß auf als im Clodronat-Arm (Abb.; Davies Fet

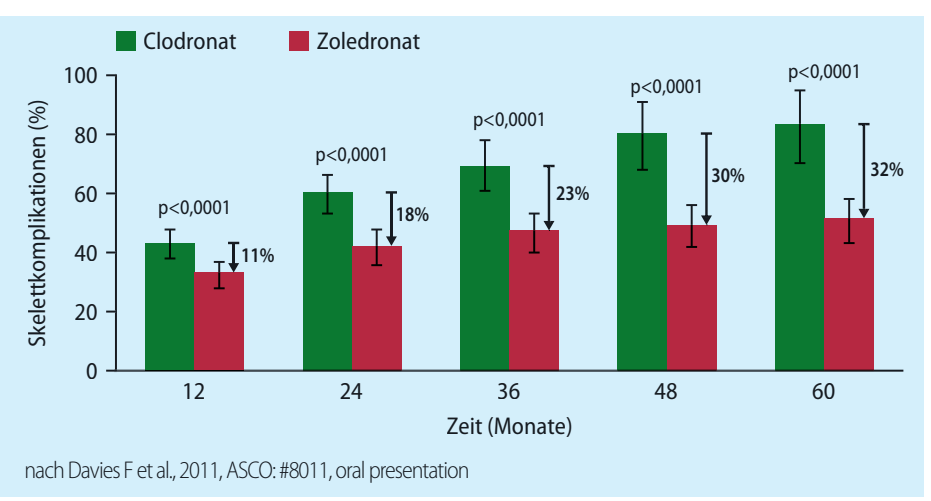

MRC-Myeloma-IX-Studie: Skelettkomplikationen unter Zoledronat versus Clodronat

al., 2011, ASCO:\#8011, oral presentation). Während bis vor Kurzem galt, eine Bisphosphonat-Therapie auf zwei Jahre zu begrenzen, sollte die Therapiedauer nun neu überdacht werden, so Goldschmidt.

Insgesamt erlitten 35,3\% der Clodronat-Patienten im Zeitraum von 42 Monaten SRE versus 27,0\% unter Zoledronat ( $p=0,0004)$. Dies entspricht einer Reduktion der SRE-Rate von 26\%. Dieser Vorteil war unabhängig davon, ob bei Studienbeginn bereits eine Knochenerkrankung vorlag oder nicht. Eine Zoledronat-Gabe auch bei Patienten mit multiplem Myelom ohne Knochenschäden scheint daher sinnvoll (Boyd K et al., 2011, ASCO: \#8010, oral presentation). CL

Quelle: Veranstaltung der Novartis Pharma GmbH

\section{Metastasiertes Mammakarzinom}

\section{Capecitabin: Hohe Wirksamkeit bei mehr Lebensqualität}

Dem in den ASCO-Guidelines formulierten Grundsatz „, Quality-adjusted Survival rather than Survival alone" entsprechend sind Mitentscheidung des Patienten, Wünsche, Erwartungen und Wertvorstellungen für die Therapiewahl wichtig, betonte PD Dr. BjörnWieland Lisboa, Duisburg.

Capecitabin ist wegen seiner gut belegten Effektivität und guten Verträglichkeit als Standard in der Therapie des metastasierten Mammakarzinoms (mMK) etabliert. Ein besonderer Vorteil des oralen Medikaments ist die nur sehr selten auftretende Alopezie, konstatierte Lisboa. Der hohe therapeutische Index konnte erneut in der Phase-IIStudie MONICA gezeigt werden: Die Zeit bis zur Tumorprogression war 7,9 Monate, das mediane Gesamtüberleben 18,6 Monate; $87 \%$ der Patientinnen vertrugen die Therapie ohne Dosismodifikation (Kaufmann M et al., 2010, Eur J Cancer 46: 3184-3191).

\section{Capecitabin-Monotherapie: Hand-Fuß- Syndrom als Hinweis auf die Wirkung? Die unter Therapie mit vielen anderen Che- motherapeutika auftretenden unerwünsch- ten Arzneimittelwirkungen, wie z.B. Neu- tropenie, febrile Neutropenie, Stomatitis etc., seien unter einer Monotherapie mit Capecitabin (Xeloda ${ }^{\oplus}$ ) so selten, dass das vergleichsweise häufiger auftretende Hand- Fuß-Syndrom (HFS) besprochen werde, er- läuterte Lisboa. Das Auftreten eines HFS könnte sogar ein Hinweis für die Wirkung sein, so die Daten der MONICA-Studie.}

\section{Capecitabin und Bevacizumab: Neue Therapieoption}

Bei hohem Remissionsdruck, d.h. bei Vorliegen multipler Organmetastasen oder bei schnell fortschreitender Metastasierung, ist der Antikörper Bevacizumab (Avastin ${ }^{\oplus}$ ) indiziert. Bevacizumab ist in Kombination mit Capecitabin für Patientinnen mit HER2-negativem mMK seit Juni 2011 zugelassen. In der randomisierten, placebokontrollierten Phase-III-Zulassungsstudie RIBBON-1 führte die Kombinationstherapie nicht zu Alopezie (Robert NJ et al., 2011, J Clin Oncol 29: 1252 1260). „Gerade in der palliativen Therapiesituation ist dieser Aspekt der Lebensqualität, der Haarerhalt, von zentraler Bedeutung", betonte Lisboa. $F H$

Quelle: Veranstaltung der Roche Pharma AG 